\title{
Repurposing the Veterinary Antibiotic Apramycin for Antibacterial and Antibiofilm Activity Against Pseudomonas aeruginosa From Cystic Fibrosis Patients
}

\author{
Giovanni Di Bonaventura ${ }^{1,2 *}$, Veronica Lupetti ${ }^{1,2}$, Fabio Verginelli ${ }^{2,3 *}$, \\ Sara Giancristofaro ${ }^{1,2,4}$, Rosemary Barbieri, ${ }^{1,2}$, Giovanni Gherardi ${ }^{5}$ and Arianna Pompilio ${ }^{2}$ \\ 'Department of Medical, Oral and Biotechnological Sciences, "G. d'Annunzio" University of Chieti-Pescara, Chieti, Italy, \\ ${ }^{2}$ Center for Advanced Studies and Technology (CAST), "G. d'Annunzio" University of Chieti-Pescara, Chieti, Italy, \\ ${ }^{3}$ Department of Pharmacy, "G. d'Annunzio" University of Chieti-Pescara, Chieti, Italy, ${ }^{4}$ Department of Infectious Disease, \\ Istituto Superiore di Sanità, Rome, Italy, ${ }^{5}$ Department of Medicine, Campus Biomedico University, Rome, Italy
}

Objectives: To evaluate the in vitro antibacterial, antibiofilm, and antivirulence activities of apramycin, comparatively to tobramycin, against a set of $P$. aeruginosa from chronically infected cystic fibrosis (CF) patients.

Methods: The activity of antibiotics against planktonic cells was assessed by performing MIC, MBC, and time-kill assays. The activity against mature biofilms was evaluated, in a microtiter plate, both in terms of dispersion (crystal violet assay) and residual viability (viable cell count). The effect of drug exposure on selected $P$. aeruginosa virulence genes expression was assessed by real-time Reverse Transcription quantitative PCR (RT-qPCR).

Results: Apramycin $\mathrm{MIC}_{90}$ and $\mathrm{MBC}_{90}$ values were found at least fourfold lower than those for tobramycin. A comparable trend was observed for mucoid strains. Only 4 out of 24 strains (16.6\%) showed an apramycin MIC higher than the epidemiological cutoff value of $64 \mathrm{mg} / \mathrm{L}$, whereas a higher resistance rate was observed for tobramycin (62.5\%; $p<0.01$ vs. apramycin). In time-kill analyses, both aminoglycosides were found bactericidal, although apramycin showed a more rapid effect and did not allow for regrowth. Apramycin generally stimulated biofilm biomass formation, whereas tobramycin showed opposite trends depending on the strain tested. Both drugs caused a highly significant, dose-dependent reduction of biofilm viability, regardless of strain and concentration tested. The exposure to apramycin and tobramycin caused increased expression of mexA and mexC (multidrug efflux pumps), whereas tobramycin specifically increased the expression of aprA (alkaline protease) and toxA (exotoxin A). Neither apramycin nor tobramycin showed cytotoxic potential toward IB3-1 bronchial epithelial CF cells.

Conclusion: Our results warrant future pharmacokinetic and pharmacodynamic studies for supporting the rationale to repurpose apramycin, a veterinary aminoglycoside, for CF lung infections.

Keywords: Pseudomonas aeruginosa, cystic fibrosis, apramycin, repurposing, biofilm 


\section{INTRODUCTION}

Cystic fibrosis (CF) is an autosomal disorder caused by a mutation in the $\mathrm{CF}$ transmembrane conductance regulator gene (CFTR), leading to an absent or dysfunctional CFTR protein with consequent alteration in the ionic transport (Cutting, 2015; Bergeron and Cantin, 2019). In the lungs, this mutation causes an accumulation of dry and sticky secretions, creating the perfect environment for bacterial infections. Among these infections, those caused by Pseudomonas aeruginosa are predominant, especially in adulthood (Maiden et al., 2018). Due to the altered microenvironment of the CF lung, inflammation fails in clearing the infection, thus causing the progression of lung disease that eventually leads to bronchiectasis and death (Chmiel et al., 2002).

Inhaled tobramycin is recommended for the eradication of P. aeruginosa infection in CF patients (Mogayzel et al., 2014; Maiden et al., 2018). However, despite intensive antibiotic therapy, the infection is virtually impossible to eradicate because of antibiotic resistance which is further aggravated by the ability of $P$. aeruginosa to grow as biofilm, an aggregation of microorganisms enclosed in an extracellular polymeric substance (EPS) and intrinsically tolerant to antimicrobials and host immune response (Hall-Stoodley et al., 2004; Keren et al., 2004; Ciofu et al., 2015; Maiden et al., 2018). In addition, as an adaptative response to the stressing $\mathrm{CF}$ airways, $P$. aeruginosa converts to a mucoid phenotype that is often associated with a poor prognosis for the CF patient, due to an overproduction of alginate and the generation of a thicker extracellular polysaccharide matrix (Folkesson et al., 2012; Maiden et al., 2018). This scenario raises the urgent need to develop new molecules to avoid selecting resistant strains and possibly target cells within sessile communities.

Apramycin is a monosubstituted deoxystreptamine exclusively used in veterinary medicine for the treatment of bovine mastitis and diarrheal disease in farm animals (European Medicines Agency [EMA], 2018). Its chemical structure differs from that of clinically relevant disubstituted aminoglycoside antibiotics, making this molecule intrinsically resilient to almost all resistance determinants typically found in multidrug-resistant (MDR) gram-negative bacteria (Wachino and Arakawa, 2012; Kang et al., 2017). Confirming this, recent studies have shown a broad-spectrum in vitro activity of apramycin against human isolates of MDR Acinetobacter baumannii, P. aeruginosa, carbapenem-resistant Enterobacteriaceae, and Staphylococcus aureus (Truelson et al., 2018; Juhas et al., 2019). In addition, apramycin has been shown to be active against lung and septicemic infections in murine models (Meyer et al., 2014; Becker et al., 2020), with lower toxicity than other disubstituted aminoglycosides (Matt et al., 2012).

Based on these compelling properties, for the first time in the present study the in vitro antibacterial, anti-biofilm, and antivirulence activities of apramycin were evaluated, comparatively to tobramycin, against a selected set of $P$. aeruginosa $\mathrm{CF}$ strains. This is a first step in assessing whether apramycin or potential derivatives of apramycin might serve as lead compounds for future therapeutics against $P$. aeruginosa infections in CF patients.

\section{MATERIALS AND METHODS}

\section{Bacterial Strains}

Twenty-four $P$. aeruginosa strains-isolated from respiratory specimens of chronically infected CF patients-were tested in the present study (Table 1). Strains resulted in being clonally distinct at PFGE analysis (Pompilio et al., 2020). Both mucoid and non-mucoid strains were enrolled since these variants are often co-isolated in CF sputum, indicating they may have selective advantages withstanding into a stressful environment such as CF lung (Clark et al., 2015; Tai et al., 2017; Malhotra et al., 2018). Furthermore, PaPh32 strain was isolated in a CF lung transplant recipient. After identification using MALDI-TOF mass spectrometry, strains were stored at $-80^{\circ} \mathrm{C}$ until use when they were cultured twice on Mueller-Hinton agar (MHA; Oxoid, Milan, Italy) to restore the original phenotypic traits (i.e., mucoid phenotype, antibiotic susceptibility, etc.).

\section{Antimicrobial Susceptibility Testing}

Apramycin and tobramycin were obtained as powder with known potency from Merck KGaA (Darmstadt, Germany). Stock solutions were prepared in reagent grade water and stored at $-80^{\circ} \mathrm{C}$ until use. The activity of drugs against planktonic $P$. aeruginosa cells was evaluated by measuring MIC (Minimum Inhibitory Concentration) and MBC (Minimum Bactericidal Concentration) values. MIC values were obtained in cationadjusted Mueller-Hinton II broth (CAMHB; Becton, Dickinson \& Co., Milan, Italy) using the broth microdilution technique and interpreted according to EUCAST guidelines (The European Committee on Antimicrobial Susceptibility Testing, 2021). Escherichia coli ATCC25922 and P. aeruginosa ATCC27853 were tested in parallel as quality control strains. $\mathrm{MBC}$ values were measured according to CLSI guidelines (National Committee for Clinical Laboratory Standards, 1999). Ten microliters of broth culture from wells showing no visible growth at MIC determination were plated onto MHA. The MBC value was defined as the minimum antibiotic concentration able to eradicate $99.9 \%$ of the starting inoculum following incubation at $37^{\circ} \mathrm{C}$ for $24 \mathrm{~h}$.

\section{Time-Kill Studies}

Time-kill assays were performed according to CLSI recommendations (National Committee for Clinical Laboratory Standards, 1999). Briefly, a standardized inoculum $\left(1-2 \times 10^{6} \mathrm{CFU} / \mathrm{mL}\right)$ prepared from an overnight growth on Tryptone Soya Agar (TSA; Oxoid, Milan, Italy) was exposed to several concentrations $(0.5 \mathrm{x}, 1 \mathrm{x}, 2 \mathrm{x}, 4 \mathrm{x}$, and $8 \mathrm{xMIC})$ of each drug prepared in CAMHB. Control samples were prepared similarly without exposure to drugs. At prefixed times of incubation at $37^{\circ} \mathrm{C}(1,2,3,4,5,6,12,16,20$, and $24 \mathrm{~h})$, aliquots were removed, and 10-fold serial dilutions were prepared in PBS for colony counting. Results were expressed by plotting Log $(\mathrm{CFU} / \mathrm{mL})$ over time, considering $10 \mathrm{CFU} / \mathrm{mL}$ as the limit of detection. Antibiotic carry-over effect was not observed. Bactericidal activity was defined as a $\geq 3 \log$ $(\mathrm{CFU} / \mathrm{mL})$ reduction. 
TABLE 1 | Susceptibility of $P$. aeruginosa CF planktonic cells.

\begin{tabular}{|c|c|c|c|c|c|}
\hline \multirow[b]{2}{*}{ Strain ID } & \multirow[b]{2}{*}{ Phenotype } & \multicolumn{2}{|c|}{ Apramycin } & \multicolumn{2}{|c|}{ Tobramycin } \\
\hline & & MIC & MBC & MIC & MBC \\
\hline $\mathrm{Pa} 7$ & & 64 & 256 & $2(\mathrm{~S})$ & 4 \\
\hline Pa37 & Mucoid & 8 & 16 & $0.25(S)$ & 0.5 \\
\hline Pa38 & Mucoid & 128 & 256 & $4(\mathrm{R})$ & 4 \\
\hline Pa39 & Mucoid & 32 & 64 & $256(R)$ & 256 \\
\hline $\mathrm{Pa} 40$ & Mucoid & 32 & 64 & $256(R)$ & 256 \\
\hline $\mathrm{Pa} 42$ & Mucoid & 16 & 32 & $0.5(\mathrm{~S})$ & 0.5 \\
\hline Pa43 & Mucoid & 8 & 16 & $0.5(\mathrm{~S})$ & 0.5 \\
\hline $\mathrm{Pa} 45$ & Mucoid & 16 & 16 & $32(R)$ & 32 \\
\hline Pa46 & Mucoid & 32 & 32 & $256(\mathrm{R})$ & 256 \\
\hline $\mathrm{Pa} 47$ & Mucoid & 16 & 32 & $64(\mathrm{R})$ & 64 \\
\hline Pa48 & & 32 & 64 & $256(R)$ & 256 \\
\hline Pa49 & & 32 & 64 & $1(\mathrm{~S})$ & 2 \\
\hline Pa50 & & 32 & 64 & $1,024(\mathrm{R})$ & $>1,024$ \\
\hline Pa51 & & 16 & 32 & $256(\mathrm{R})$ & 256 \\
\hline Pa52 & & 32 & 64 & $1(\mathrm{~S})$ & 2 \\
\hline Pa53 & Mucoid & 16 & 16 & $1(\mathrm{~S})$ & 2 \\
\hline Pa54 & & 8 & 16 & $32(\mathrm{R})$ & 64 \\
\hline Pa55 & & 512 & 512 & $>1,024(\mathrm{R})$ & $>1,024$ \\
\hline Pa56 & & 16 & 16 & $0.5(\mathrm{~S})$ & 1 \\
\hline Pa57 & & 128 & 128 & $512(\mathrm{R})$ & 1,024 \\
\hline PaPh16 & & 64 & 128 & $512(\mathrm{R})$ & 512 \\
\hline PaPh26 & & 128 & 256 & $8(\mathrm{R})$ & 8 \\
\hline PaPh27 & & 8 & 8 & $0.5(\mathrm{~S})$ & 0.5 \\
\hline PaPh32 & Mucoid & 32 & 64 & $64(\mathrm{R})$ & 128 \\
\hline
\end{tabular}

MIC and MBC values were measured by the broth microdilution method and expressed as $\mathrm{mg} / \mathrm{L}$. In brackets, the categorical interpretations of susceptibility $(S)$ and resistance (R) for tobramycin, considering a susceptibility breakpoint of $\leq 2 \mathrm{mg} / \mathrm{L}$ (The European Committee on Antimicrobial Susceptibility Testing, 2021).

\section{Screening for Biofilm Formation}

All strains were screened for their ability to form biofilm using the microtiter plate method. A bacterial suspension-grown overnight in Trypticase Soy broth (TSB; Oxoid) at $37^{\circ} \mathrm{C}$ and under dynamic conditions (130 rpm)-was adjusted with sterile TSB to an optical density measured at $550 \mathrm{~nm}\left(\mathrm{OD}_{550}\right)$ equals to 1.0 (corresponding to $1-4 \times 10^{8} \mathrm{CFU} / \mathrm{mL}$ ) and diluted $1: 100$ ( $\mathrm{vol} / \mathrm{vol}$ ) using sterile TSB. Two hundred microliters of this standardized inoculum were added to each well of a 96-well polystyrene tissue culture plate (Falcon BD; Becton, Dickinson \& Co.). Negative controls were prepared similarly without adding the standardized inoculum. After static $24 \mathrm{~h}$-incubation at $37^{\circ} \mathrm{C}$, biofilms were washed twice with PBS (pH 7.2) (Merck $\mathrm{KGaA})$, fixed $\left(60^{\circ} \mathrm{C}\right.$ for $\left.1 \mathrm{~h}\right)$, stained with Hucker-modified crystal violet (5 $\mathrm{min}, 200 \mu \mathrm{L} /$ well) (Sonnenwirth, 1980), and air-dried $\left(37^{\circ} \mathrm{C}, 30 \mathrm{~min}\right)$. Crystal violet was extracted using $33 \%$ glacial acetic acid (Merck KGaA) (15 min, $200 \mu \mathrm{L} /$ well). Finally, biofilm biomass was quantified spectrophotometrically, measuring $\mathrm{OD}_{492}$ (Sunrise; Tecan, Milan, Italy) (Pompilio et al., 2020). The percentage of biofilm dispersion caused by drug exposure was calculated as follows: $\left(1-\mathrm{OD}_{492}\right.$ of test/OD 492 of untreated control $) \times 100$. According to the criteria proposed by Stepanović et al. (2007) a strain was classified for biofilm formation as follows: non-producer $\left(\mathrm{OD} \leq \mathrm{OD}_{c}\right)$; weakproducer $\left[\mathrm{OD}_{c}<\mathrm{OD} \leq\left(2 \times \mathrm{OD}_{c}\right)\right]$; moderate-producer $[(2 \times$ $\left.\left.\mathrm{OD}_{c}\right)<\mathrm{OD} \leq\left(4 \times \mathrm{OD}_{c}\right)\right]$; or strong-producer $\left(\mathrm{OD}>4 \times \mathrm{OD}_{c}\right)$. The cut-off value $\left(\mathrm{OD}_{c}\right)$ was defined as the mean $\mathrm{OD}$ of negative control $+3 \times$ SDs.

\section{In vitro Activity Against Preformed Biofilm}

Twenty-four hour old biofilms formed in a 96-well microtiter plate as described in "Screening for biofilm formation" were exposed to each drug tested at the desired concentrations in CAMHB. Following $24 \mathrm{~h}$-exposure at $37^{\circ} \mathrm{C}$ under static conditions, the effect against mature biofilm was evaluated in terms of dispersion-using crystal violet assay, as described above-and residual viability by viable cell count. In the latter, biofilms were washed twice with sterile PBS to eliminate the drug and not attached cells, then exposed for $5 \mathrm{~min}$ to $200 \mu \mathrm{L}$ trypsinethylenediaminetetraacetic acid $0.25 \%$ (Merck KGaA). Detached cells were collected by manual scraping and finally underwent viable cell count onto TSA. The percentage of biofilm viability after drug exposure was calculated as follows: [(CFU/well of test $) /(\mathrm{CFU} /$ well of untreated control) $] \times 100$.

\section{Gene Expression Assay}

The effect of drug exposure on the transcription levels of algD, toxA, lasI, aprA, mexA, mexB, and mexC virulence genes was assessed by real-time Reverse Transcription quantitative PCR (RT-qPCR). Planktonic cells were exposed to each drug at $0.25 \mathrm{xMIC}$ for $20 \mathrm{~h}$ at $37^{\circ} \mathrm{C}$, washed with PBS, and then directly harvested in Qiazol Lysis Reagent (Qiagen; Milan, Italy), a monophasic solution of phenol and guanidine thiocyanate designed to facilitate lysis and inhibit RNases. RNA was extracted following the manufacturer's protocol adding chloroform for isolation followed by isopropanol and ethanol washes for purification. After DNase I treatment (Merck KGaA), RNA was checked for purity and quantity by NanoDrop-2000 (Thermo Fisher Scientific Italia Inc., Milan, Italy). RNA quality was assessed by running an aliquot of samples on a denaturing agarose gel stained with ethidium bromide. First-strand cDNA was synthesized from $2 \mu \mathrm{g}$ of RNA using a High-Capacity cDNA reverse transcription kit (Thermo Fisher Scientific Italia Inc.). Next, gene expression was evaluated using $10 \mathrm{ng}$ cDNA by real-time RT-qPCR assay on QuantStudio ${ }^{\mathrm{TM}} 7$ Pro RealTime PCR System (Applied Biosystems) using the PowerTrack SYBR Green Master Mix (Thermo Fisher Scientific Italia Inc.). Primers were designed using as a reference the genome of P. aeruginosa strain NDTH9845 (GeneBank accession number: CP073080.1) (Supplementary Table 1). Considering that the difference between the melting temperature (Tm) and the annealing temperature (Ta) should be $\leq 5^{\circ} \mathrm{C}$, all oligonucleotides were designed with a Tm not exceeding $65^{\circ} \mathrm{C}$ to use a Ta $=60^{\circ} \mathrm{C}$ for all amplifications. Specificity was assessed both in silico with BLAST and by PCR endpoint under the same real-time RTqPCR conditions. The $\Delta \Delta \mathrm{Ct}$ method was applied to determine the relative gene expression in exposed vs. unexposed cells after 
normalizing on the expression of the proC housekeeping gene. The modulation of expression levels was shown as fold change on a $\log _{2}$ scale.

\section{Cytotoxicity Evaluation}

The direct cytotoxic effect of each drug was assessed toward IB3-1 bronchial epithelial cells (ATCC\#CRL-2777) isolated from a pediatric CF patient who harbored the $\triangle \mathrm{F} 508 / \mathrm{W} 1282 \mathrm{X}$ mutations within the CFTR gene. Cells were grown as monolayer at $37^{\circ} \mathrm{C}$ in LHC-8 medium (Thermo Fisher Scientific Italia Inc.) supplemented with $5 \%$ fetal bovine serum (Thermo Fisher Scientific Italia Inc.) in a $5 \% \mathrm{CO}_{2}$ atmosphere. After exposing the monolayer to each drug at the desired concentration for $24 \mathrm{~h}$, the cell viability was measured by an MTS tetrazoliumbased colorimetric assay (CellTiter $96^{\circledR}$ AQueous One Solution Cell Proliferation Assay, Promega, Milan, Italy). Briefly, 20 $\mu \mathrm{L}$ of a mixture of MTS [3-(4,5-dimethylthiazol-2-yl)-5-(3carboxymethoxyphenyl)-2-(4-sulfophenyl)-2H-tetrazolium] and the electron coupling reagent PES (phenazine ethosulfate) were added to each well containing exposed cells. Untreated IB3-1 cells were prepared as the control. After $4 \mathrm{~h}$-incubation at $37^{\circ} \mathrm{C}$, the $\mathrm{OD}_{492}$ was measured using an ELISA plate reader (Sunrise, Tecan Trading AG, Switzerland).

\section{Statistical Analysis}

Each experiment was carried out at least in triplicate and repeated on two different occasions $(n \geq 6)$. Statistical analysis was performed using GraphPad software (ver. 8.0; GraphPad Inc., CA, United States). Data distribution was assessed using the D'Agostino \& Pearson normality test, and then the statistical significance of differences was evaluated using: (i) ordinary one-way ANOVA followed by Dunnett's multiple comparisons post-test, for datasets normally distributed; (ii) Holm-Sidak's or Tukey's multiple comparisons post-test in case datasets did not pass the normality test. Differences between percentages were evaluated using Fisher's exact test. The significance level was set at $p<0.05$. Differences between MIC or MBC values were considered as significant for discrepancies $\geq 2 \log _{2}$ concentration steps.

\section{RESULTS AND DISCUSSION}

MIC and MBC values for apramycin and tobramycin toward $24 P$. aeruginosa CF strains were obtained using the broth microdilution technique, and results are shown in Table $\mathbf{1}$. A high degree of resistance to tobramycin was observed (15 out of 24 strains, $62.5 \%$ ). Although it was not possible to make a similar categorical assessment for apramycin due to the lack of established clinical EUCAST/CLSI breakpoints, MIC $_{90}$ and $\mathrm{MBC}_{90}$ values (i.e., the lowest concentration of an antimicrobial capable to inhibit or kill $90 \%$ of bacterial isolates, respectively) were significantly lower, at least 4-8-fold, than tobramycin (128 vs. $1,024 \mathrm{mg} / \mathrm{L}$, and 256 vs. $1,024 \mathrm{mg} / \mathrm{L}$, respectively). Our findings are concordant with a previous study where the activity of apramycin against MDR A. baumannii and P. aeruginosa clinical isolates was evaluated (Kang et al., 2017).
A similar trend was observed when considering mucoid strains only, with apramycin $\mathrm{MIC}_{90}$ and $\mathrm{MBC}_{90}$ values, respectively, 8- and 4-fold lower than tobramycin (32 vs. $256 \mathrm{mg} / \mathrm{L}$, and 64 vs. $256 \mathrm{mg} / \mathrm{L}$, respectively). This might be particularly relevant in CF patients where $P$. aeruginosa mucoid conversion is associated with a worse prognosis (Li et al., 2005; Malhotra et al., 2019).

In accordance with previous evidence (Kang et al., 2017), the visual inspection of apramycin MIC distribution suggested an epidemiological cut-off value of $64 \mathrm{mg} / \mathrm{L}$ (Figure 1). It is worth noting that only 4 out of $24(16.6 \%)$ strains had an MIC above this cut-off suggesting very low levels of acquired apramycin resistance, significantly lower than that observed for tobramycin ( 15 out of $24,62.5 \% ; p<0.01$ vs. apramycin). Overall, these findings suggest the intrinsic resilience of apramycin to common mechanisms of aminoglycoside resistance (Davies and O'Connor, 1978; Lovering et al., 1987), in this highly tobramycin-resistant $P$. aeruginosa strain set. Furthermore, no cross-resistance occurred (relationship between MIC values of two drugs; Pearson correlation coefficient r: 0.169). Comparative analysis of $\mathrm{MBC}$ and $\mathrm{MIC}$ values performed by calculating the killing quotient ( $\mathrm{KQ}=\mathrm{MBC} / \mathrm{MIC}$ ) indicated that both aminoglycosides are bactericidal $(\mathrm{KQ}<4)$ against all strains tested. The bactericidal effect of apramycin was confirmed by time-to-kill assays performed on $\mathrm{PaPh} 32$ and $\mathrm{Pa} 7$ strains, respectively, representative for tobramycin-resistant and susceptible phenotypes (Figure 2). Both aminoglycosides showed dose-dependent bactericidal activity, although to different extents. Indeed, although apramycin and tobramycin showed comparable time-kill kinetic toward $\mathrm{Pa} 7$ strain-resulting bactericidal at $8 \mathrm{x}, 4 \mathrm{x}$, and $2 \mathrm{xMIC}$-apramycin resulted more

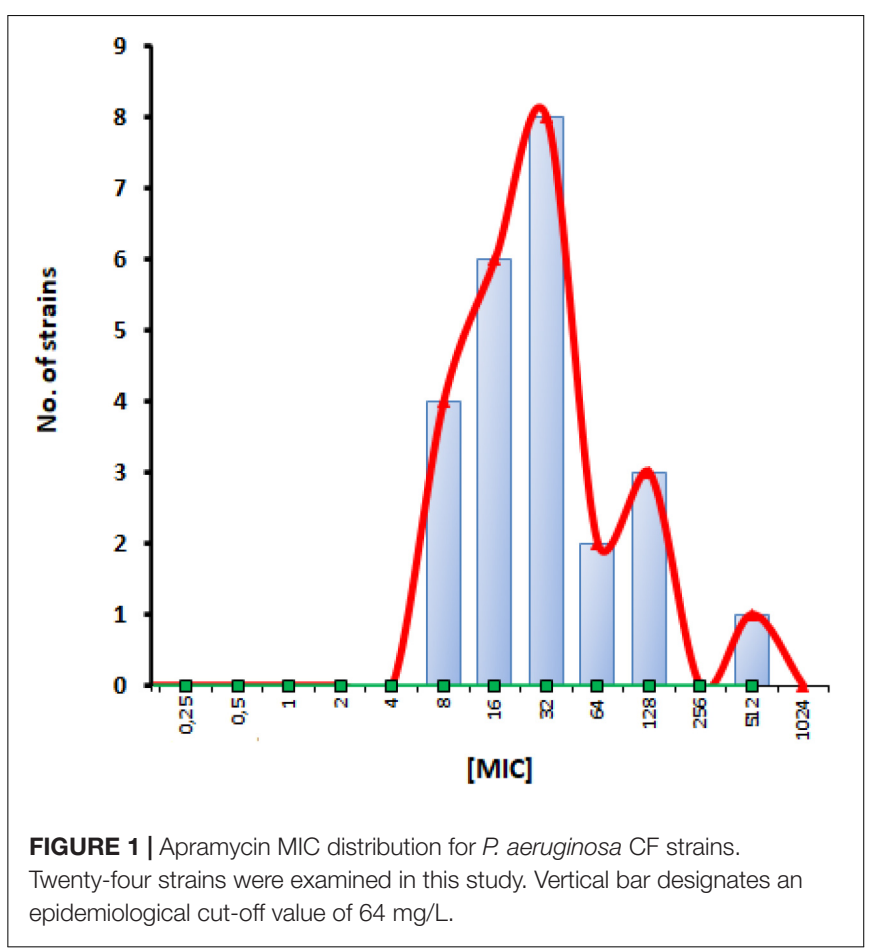




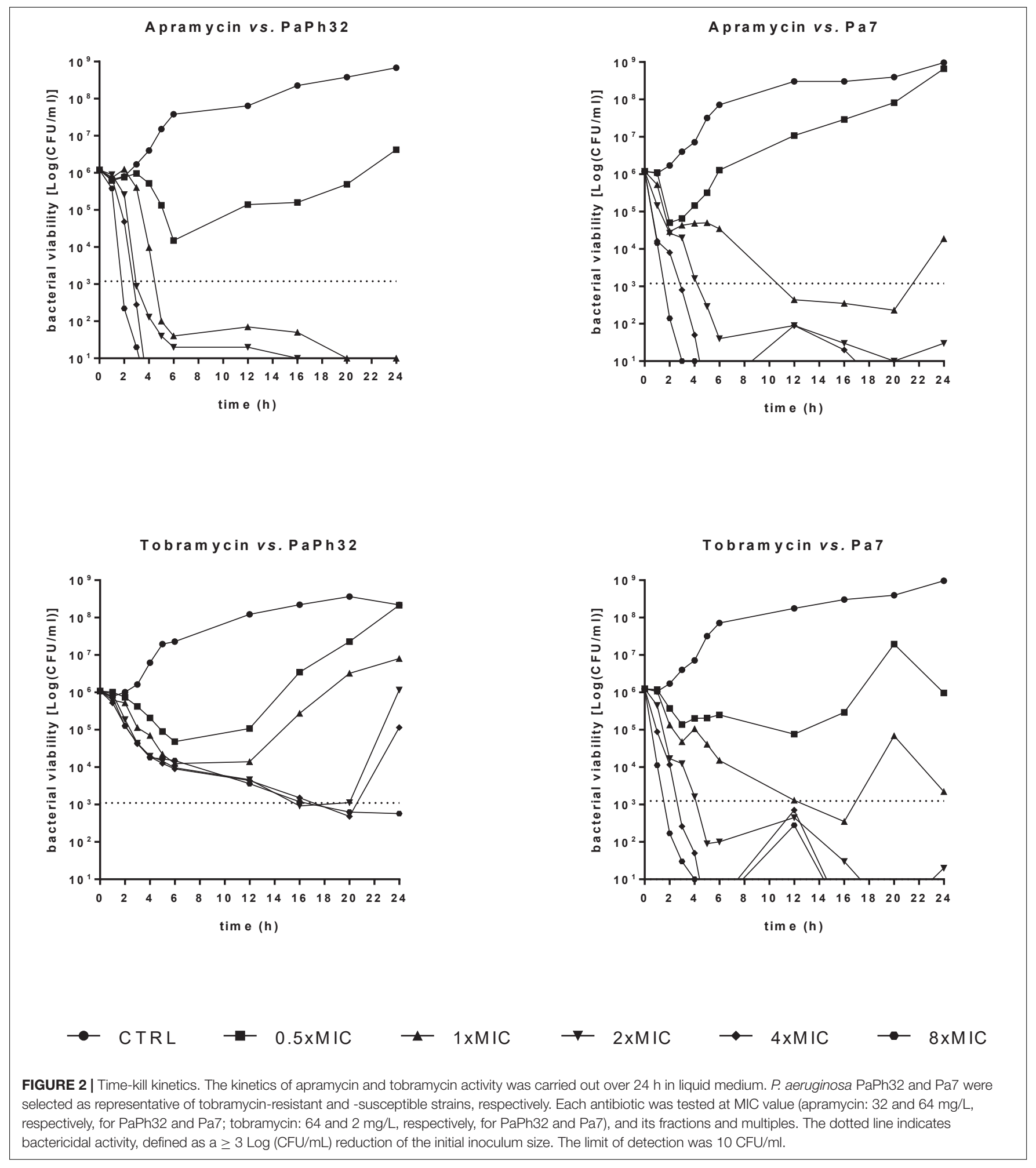

effective against the tobramycin-resistant $\mathrm{PaPh} 32$ strain, proving to be bactericidal already after 2 h-exposure at $8 x M I C$, and within 6 h-exposure at $4 x, 2 x$, and 1xMIC. Conversely, tobramycin exerted bactericidal effect only at $8 \mathrm{x}$ and $4 \mathrm{xMIC}$, although a regrowth was observed at $4 \mathrm{xMIC}$. These findings are consistent with prior observations of rapid bactericidal activity of apramycin toward N. gonorrhoeae (Riedel et al., 2019), A. baumannii (Kang et al., 2018), and S. aureus (Truelson et al., 2018), whereas no data has been published for $P$. aeruginosa. Overall, our data indicate that apramycin might be preferable 


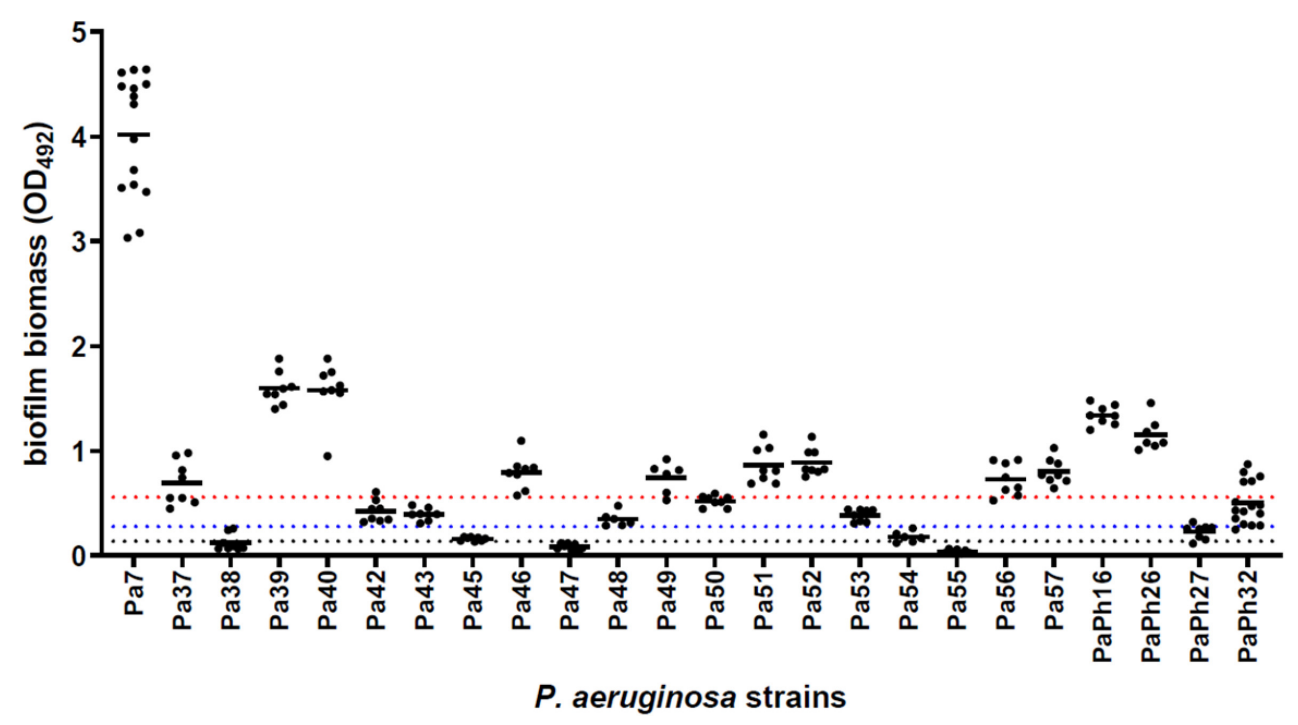

FIGURE 3 | Biofilm formation by P. aeruginosa strains from CF patients. The amount of biofilm formed following $24 \mathrm{~h}$-incubation at $37^{\circ} \mathrm{C}$ was measured in polystyrene 96-well microtiter plate using the crystal violet assay. Results are shown as scatter plot, with the horizontal solid line indicating the mean OD value. According to the criteria proposed by Stepanović et al. (2007) a strain was classified for biofilm formation as follows: non-producer (OD $\leq \mathrm{OD}_{C}$; below the dotted black line); weak-producer $\left[\mathrm{OD}_{c}<\mathrm{OD} \leq\left(2 \times \mathrm{OD}_{C}\right)\right.$; between dotted black and blue lines]; moderate-producer $\left[\left(2 \times \mathrm{OD}_{C}\right)<\mathrm{OD} \leq\left(4 \times \mathrm{OD}_{C}\right)\right.$; between dotted blue and red lines]; or strong-producer $\left(\mathrm{OD}>4 \times \mathrm{OD}_{c}\right.$; above the dotted red line). Cut-off value $\left(\mathrm{OD}_{c}\right)$ was defined as the mean $\mathrm{OD}$ of negative control $+3 \times \mathrm{SDs}$.

to tobramycin for rapid, early, time-kill properties against P. aeruginosa.

Chronic $P$. aeruginosa CF lung infections evolve to generate environmentally adapted clusters of communities, so-called biofilms, suspended within the airway mucus and inherently resistant to antibiotics and innate host defenses, thus leading to increased morbidity and mortality (Høiby et al., 2010; Alhede et al., 2011). According to the criteria proposed by Stepanović et al. (2007) most strains (21 out of $24,87.5 \%$ ) could be classified as biofilm producers, and $50 \%$ as strong producers (Figure 3 ), thus confirming that the biofilm mode of life is relevant to the persistence of $P$. aeruginosa during long-term colonization of CF airways (Rossi et al., 2021).

In this frame, apramycin and tobramycin were tested for the potential to disperse and kill mature biofilm by $P$. aeruginosa. To this end, Pa7 (tobramycin-susceptible, the strongest biofilm producer, frayed phenotype) and $\mathrm{PaPh} 32$ (tobramycin-resistant, moderate biofilm producer, mucoid phenotype) strains were chosen to evaluate the antibiofilm activity dependence on the susceptibility to tobramycin and the amount of biofilm formed. Results from biofilm dispersal assays indicated that the exposure to apramycin generally stimulates the formation of biofilm biomass, consisting of both cells and self-produced EPS, although at different extent depending on strain and concentration considered (Figure 4). Indeed, although lower concentrations seemed to be more effective in stimulating biofilm formation, exposure to apramycin at 8xMIC provoked a significant dispersion of biofilm formed by PaPh32 (OD 492 , mean \pm SD: $0.186 \pm 0.008$ vs. $0.465 \pm 0.05$, respectively, for exposed and unexposed biofilms; $p<0.05$ ). On the contrary, tobramycin caused a significant reduction of biofilm biomass formed by the Pa7 strain regardless of concentration. An opposite trend was observed for $\mathrm{PaPh} 32$ strain whose biofilm amount resulted to be significantly increased after exposure to tobramycin at $0.5 x M I C\left(\mathrm{OD}_{492}\right.$, mean $\pm \mathrm{SD}: 0.633 \pm 0.079$ vs. $0.465 \pm 0.05$, respectively, for treated and control biofilms; $p<0.01$ ), whereas a significant reduction was found after exposure at $8 \mathrm{xMIC}\left(\mathrm{OD}_{492}\right.$, mean $\pm \mathrm{SD}: 0.263 \pm 0.022$ vs. $0.465 \pm 0.05$, respectively, for treated and control biofilms; $p<0.01$ ). Tobramycin did not exert any significant effect when tested at $1 \mathrm{x}, 2 \mathrm{x}$, and $4 \mathrm{xMIC}$.

Next, the effect of antibiotics at multiples of MIC on the viability of preformed biofilm was evaluated by viable cell count assay, and results are summarized in Figure 5. Both drugs caused a highly significant, dose-dependent reduction of biofilm viability, regardless of strain tested. Particularly, when tested at $4 \mathrm{x}$ and $8 \mathrm{xMIC}$ against $\mathrm{Pa} 7$ mature biofilm, apramycin and tobramycin showed a killing rate $>99.9 \%$ (Figures 5A,B). Considered as a whole, our findings indicate that both antibiotics are effective in significantly reducing the viability of preformed biofilm, whereas stimulate EPS production in a strain-dependent manner.

The rise in antibiotic resistance made urgently necessary the exploitation of alternative antibacterial strategies, such as antivirulence therapy (Kang et al., 2021). This approach might be useful for $P$. aeruginosa whose extensive repertoire of virulence factors, along with its adaptability, facilitate its persistence into the hostile environment of the CF airways (Jurado-Martín et al., 2021; Rossi et al., 2021). In this frame, the effect of apramycin on the expression of selected $P$. aeruginosa virulence genes (aprA, lasI, mexA, mexB, mexC, toxA, and $\operatorname{alg} D)$ was evaluated comparatively to tobramycin, and results are resumed in 


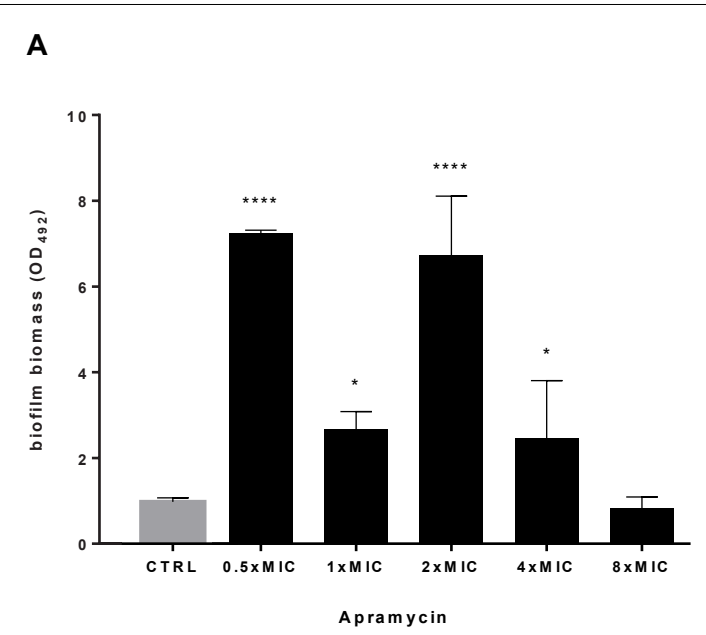

C

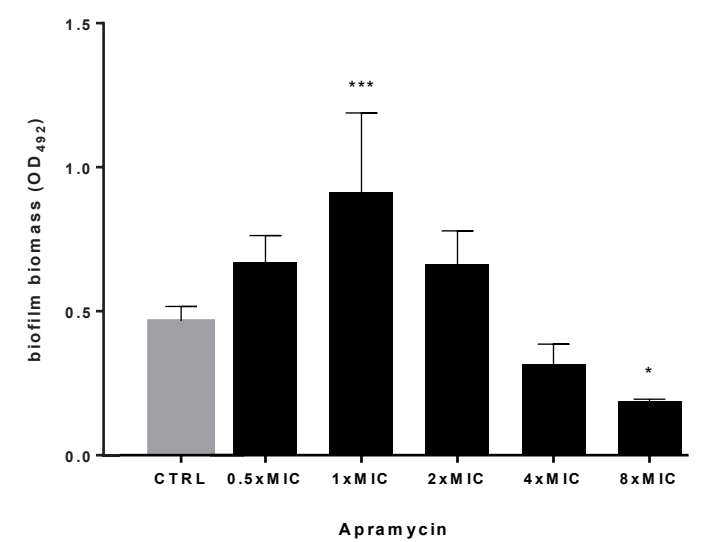

B

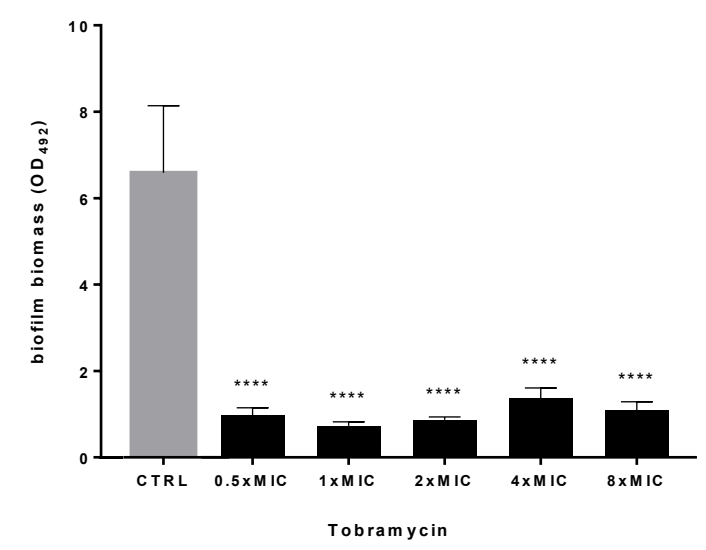

D

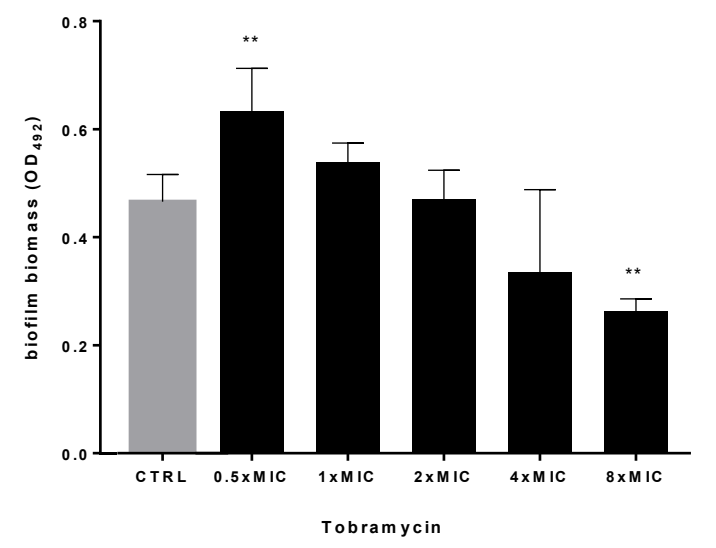

FIGURE 4 | Dispersal activity against preformed $P$. aeruginosa biofilm. The efficacy of apramycin and tobramycin to disperse 24 h-mature biofilm by $P$. aeruginosa $\mathrm{Pa} 7(\mathbf{A}, \mathbf{B})$ and PaPh32 (C,D) was assessed using crystal violet assay. Each drug was tested at fractions and multiples of MIC value (apramycin: 64 and 32 mg/L; tobramycin: 2 and 64 mg/L, respectively, for Pa7 and PaPh32). Results are expressed as mean + SD of the residual biofilm biomass (OD 492$)$ after 24 h-exposure. Control samples (CTRL) were not exposed to drug. Statistical significance at ordinary one-way ANOVA followed by Holm-Sidak's multiple comparisons post-test: ${ }^{\star} p<0.05,{ }^{\star *} p<0.01,{ }^{\star * \star} p<0.001,{ }^{* \star * \star} p<0.0001$ vs. CTRL.

Figure 6. A hallmark of $P$. aeruginosa isolates causing CF chronic infections is the multidrug resistance, mainly due to the presence of multidrug efflux (Mex) pumps (Lister et al., 2009; Llanes et al., 2011). Here we observed that both apramycin and tobramycin increase the expression of mexA (fold-change, mean $\pm \mathrm{SD}$ : $1.596 \pm 0.271$ for apramycin, $p<0.05$ vs. CTRL; $1.589 \pm 0.148$ for tobramycin, $p<0.001$ vs. CTRL), and mexC (fold-change: $3.569 \pm 0.647$ for apramycin, $p<0.001$ vs. CTRL; $5.258 \pm 0.038$ for tobramycin, $p<0.001$ vs. CTRL), theoretically conferring cross-resistance to a broad range of antibiotics, such as betalactams, chloramphenicol, tetracycline, macrolides, novobiocin, fluoroquinolones, sulfamethoxazole, and trimethoprim (Masuda et al., 2000; Morita et al., 2001).
Of note, we observed that exposure to tobramycin might specifically raise the virulence potential in $P$. aeruginosa as suggested by the increased expression of aprA (fold-change: $2.211 \pm 0.146, p<0.001$ vs. CTRL) and toxA (foldchange: $2.500 \pm 0.071, p<0.001$ vs. CTRL), respectively, encoding for alkaline protease and exotoxin A. Alkaline protease is a zinc-dependent metallo-endopeptidase involved in phagocytic evasion, reducing mucocilliary bacterial clearance, and preventing flagellin-mediated immune recognition (JuradoMartín et al., 2021). Exotoxin A, an ADP-ribosyl transferase inactivating protein synthesis (Michalska and Wolf, 2015), plays a role in establishing and the persistence of $P$. aeruginosa infection (Gallant et al., 2000). In CF patients it also stimulates 


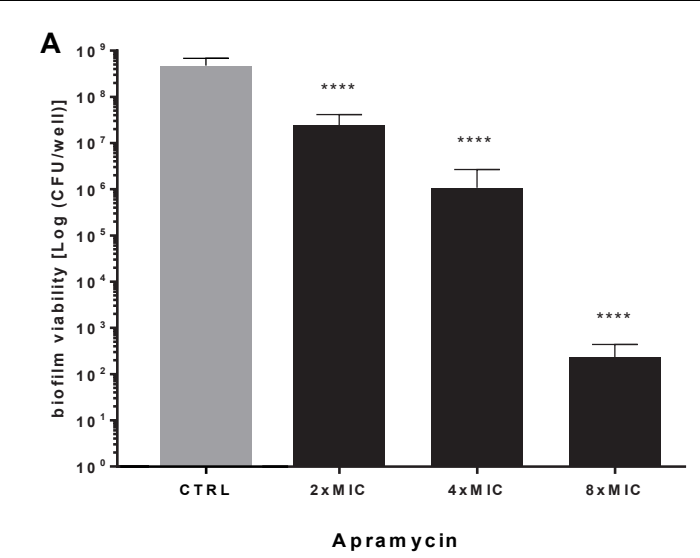

C

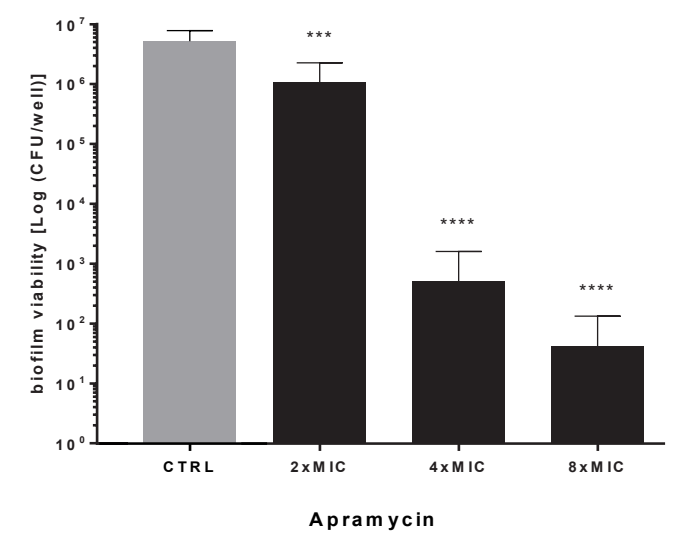

B

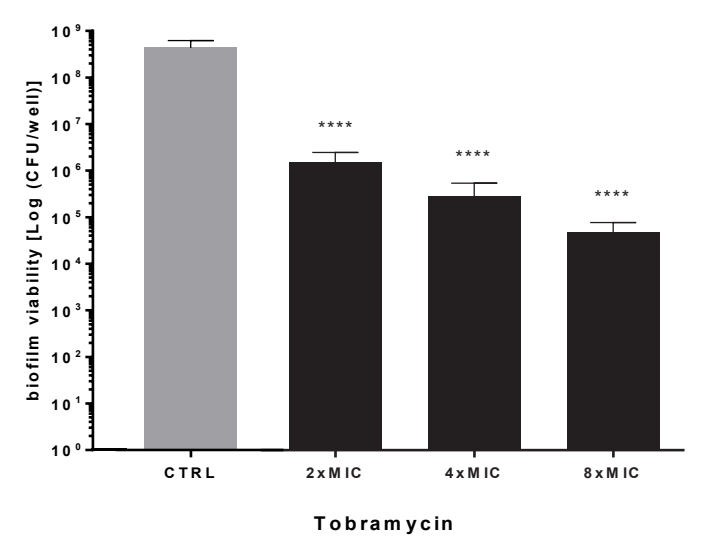

D

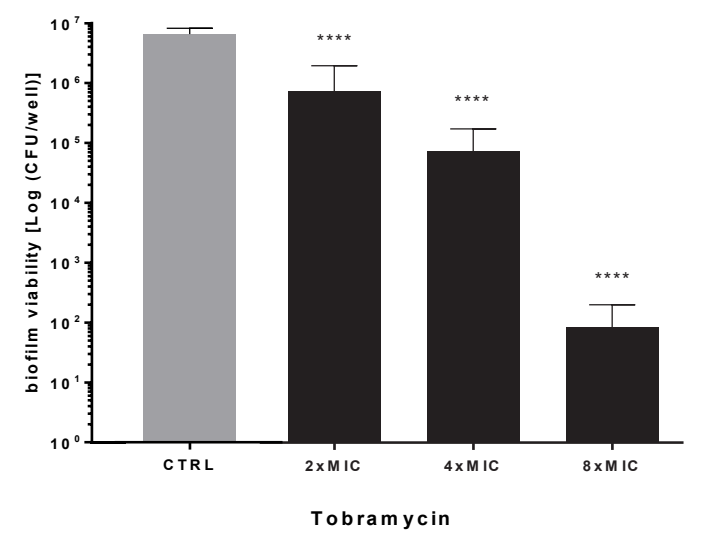

FIGURE 5 | Killing activity against preformed $P$. aeruginosa biofilm. The efficacy of apramycin and tobramycin on the viability of 24 h-mature biofilm by $P$. aeruginosa $\mathrm{Pa} 7(\mathbf{A}, \mathbf{B})$ and PaPh32 (C,D) was assessed by viable cell count assay. Each drug was tested at multiples of MIC value. Results are expressed as mean $+\mathrm{SD}$ of the residual biofilm viability [Log (CFU/well)] after 24 h-exposure. Control samples (CTRL) were not exposed to drug. Statistical significance at ordinary one-way ANOVA followed by Holm-Sidak's multiple comparisons post-test: ${ }^{\star * *} p<0.001,{ }^{\star * \star *} p<0.0001$ vs. CTRL.

epithelial cells and alveolar macrophages to produce group IIA secreted phospholipase A2 that selectively kills S. aureus, thus allowing P. aeruginosa to be progressively predominant in adult patients' airways (Belmadi et al., 2018).

The exposure to apramycin and tobramycin specifically caused $\operatorname{alg} D$ down expression in $P$. aeruginosa, although we observed statistical significance only in the case of tobramycin (fold-change: $-1.441 \pm 0.023, p<0.01$ vs. CTRL). This finding might be of relevance in the management of CF patients where the transition from acute to chronic lung infection is driven by the emergence of isolates showing a mucoid phenotype due to the overproduction of alginate codified by the operon $\operatorname{algD}$ (Bianconi et al., 2015). Indeed, alginate-overproducer $P$. aeruginosa strains promote the biofilm lifestyle and are therefore associated with poor prognosis and increased mortality (Smith et al., 2013; Malhotra et al., 2019).
The expression of other genes tested, lasI and mexB, were never significantly affected, regardless of antibiotic considered.

The use of aminoglycosides has been severely curtailed by important side effects, mainly ototoxicity and nephrotoxicity (Forge and Schacht, 2000). Recent in-vitro and in-vivo studies have indicated lower ototoxicity of apramycin, with absence of hidden hearing loss (Matt et al., 2012; Ishikawa et al., 2019), due to its exquisite selectivity for the bacterial over the eukaryotic cytosolic and mitochondrial ribosomes (Matt et al., 2012).

In this frame, evaluating the potential of apramycin for the treatment of CF lung infections requires assessing its cytotoxic potential toward the airway's epithelium. For the first time in literature, the toxic potential of apramycin toward IB3-1 bronchial epithelial CF cells was assessed using a cell-based MTS assay. The exposure to apramycin and tobramycin at $512 \mathrm{mg} / \mathrm{L}-$ the highest concentration causing significant bactericidal and 


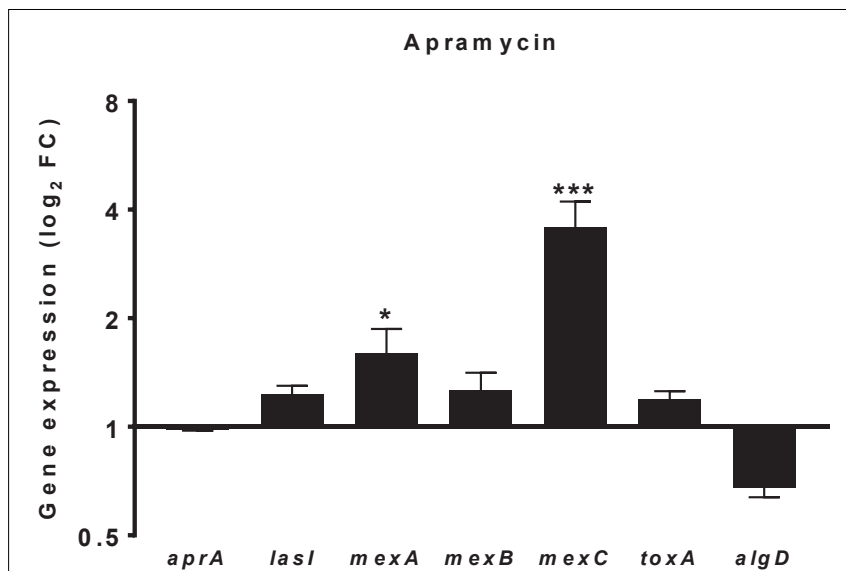

Tobram ycin

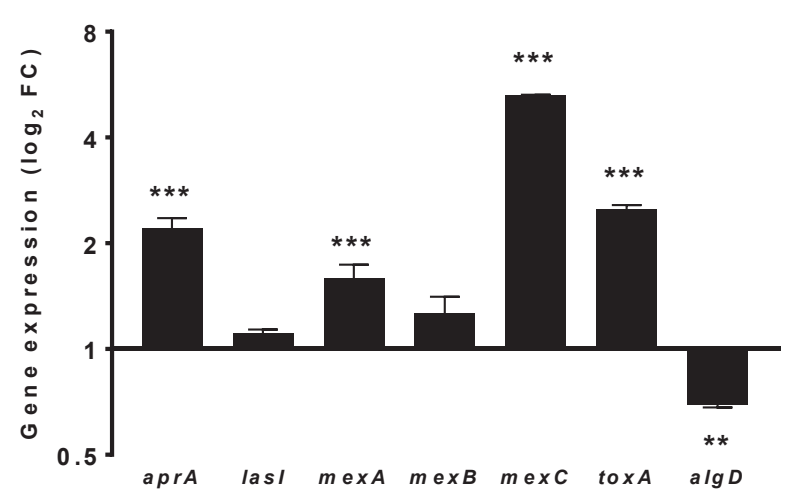

FIGURE 6 | Effect of drug exposure on the expression of $P$. aeruginosa virulence genes. The effect of $20 \mathrm{~h}$-exposure of apramycin and tobramycin at $0.25 \times \mathrm{MIC}$ on the expression of aprA (alkaline protease), lasl (quorum sensing mediator), mex $A-B-C$ (multidrug efflux pumps), tox $A$ (exotoxin $A$ ), and alg $D$ (alginate synthesis) by $P$. aeruginosa PaPh32 was measured using the real-time RT-qPCR technique. Control samples (CTRL) were not exposed to drug. The relative expression of each gene was normalized to that of the housekeeping gene (proC). Results are presented as fold change (FC: $2^{-\Delta \Delta c t}$, mean $\pm S D$ ) on a $\log _{2}$ scale. Statistical significance at ordinary one-way ANOVA followed by Tukey's multiple comparisons post-test: ${ }^{*} p<0.05,{ }^{* *} p<0.01$, and ${ }^{* * *} p<0.001$ vs. CTRL.

antibiofilm effects-did not cause any damage toward IB31 cells, whose growth was comparable to untreated control cells (Figure 7).

Despite the preliminary nature of our findings, we believe that apramycin may be worthy of consideration for repurposing in CF patients. Indeed, the rapid bactericidal activity and the low risk for acquired resistance we observed, along with the putative absence of typical aminoglycoside-associated ototoxicity reported earlier, point out the potential of apramycin, either directly and/or after derivatization, for development as an alternative treatment of $P$. aeruginosa infections. The efficacy against mature biofilms and the lack of toxicity toward CF bronchial cells are shared by apramycin and tobramycin. However, the higher activity exhibited by apramycin against mucoid strains

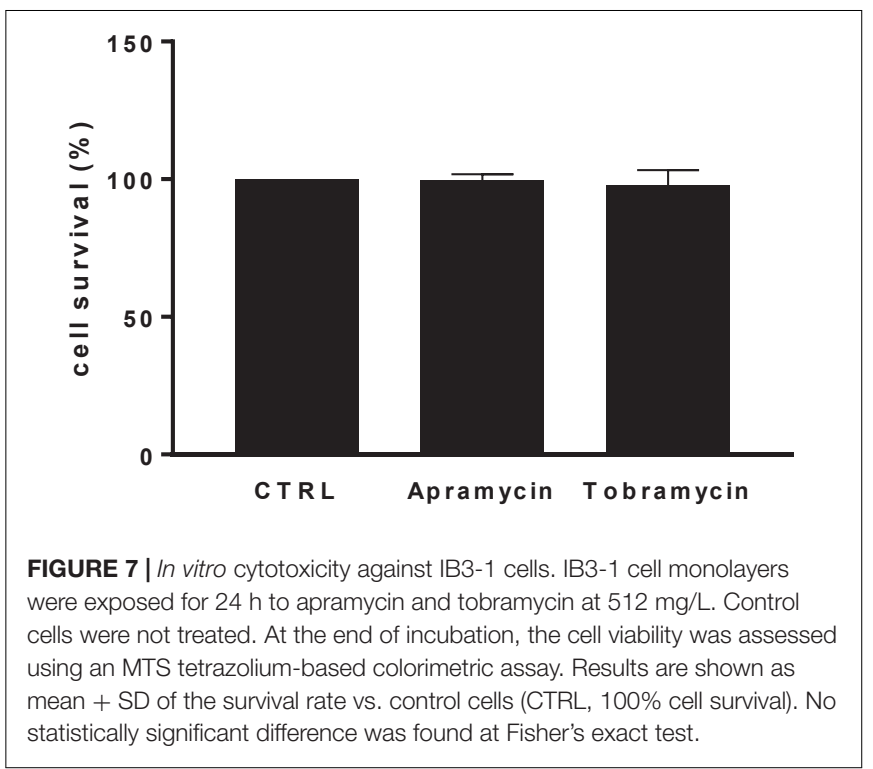

along with the increased $P$. aeruginosa virulence following tobramycin exposure might make apramycin preferable in CF patients. Further human preclinical pharmacokinetic and pharmacodynamic studies are warranted to assess the clinical potential of our findings.

\section{DATA AVAILABILITY STATEMENT}

The original contributions presented in the study are included in the article/Supplementary Material, further inquiries can be directed to the corresponding author/s.

\section{AUTHOR CONTRIBUTIONS}

GD and AP: substantial contributions to the conception or design of the work, the acquisition, analysis, and interpretation of data for the work. GD, AP, FV, and GG: drafting the work and revising it critically for important intellectual content, provide approval for publication of the content. SG, VL, AP, FV, and RB: performing experimental activity. All authors contributed to the article and approved the submitted version.

\section{FUNDING}

The research leading to these results has been supported by the "G. d'Annunzio" University of Chieti-Pescara ("F.A.R.2020" grant, assigned to GD, FV, and AP).

\section{ACKNOWLEDGMENTS}

$P$. aeruginosa strains have been kindly provided by Ersilia Fiscarelli-Cystic Fibrosis Diagnostics, Microbiology, and 
Immunology Diagnostics, "Bambino Gesù" Children's Hospital, Rome, Italy. We would like to thank Sherry Lynn Jones-International Critical Stress Foundation, Ellicott City, Maryland, United States-for proofreading the manuscript.

\section{REFERENCES}

Alhede, M., Kragh, K. N., Qvortrup, K., Allesen-Holm, M., van Gennip, M., Christensen, L. D., et al. (2011). Phenotypes of non-attached Pseudomonas aeruginosa aggregates resemble surface attached biofilm. PLoS One 6:e27943. doi: 10.1371/journal.pone.0027943

Becker, K., Aranzana-Climent, V., Cao, S., Nilsson, A., Shariatgorji, R., Haldimann, K., et al. (2020). Efficacy of EBL-1003 (apramycin) against Acinetobacter baumannii lung infections in mice. Clin. Microbiol. Infect. 27, 1315-1321. doi: 10.1016/j.cmi.2020.12.004

Belmadi, N., Wu, Y., and Touqui, L. (2018). Immuno-modulatory functions of the type- 3 secretion system and impacts on the pulmonary host defense: a role for ExoS of Pseudomonas aeruginosa in cystic fibrosis. Toxicon 143, 68-73. doi: 10.1016/j.toxicon.2018.01.004

Bergeron, C., and Cantin, A. M. (2019). Cystic fibrosis: pathophysiology of lung disease. Semin. Respir. Crit. Care Med. 40, 715-726.

Bianconi, I., Jeukens, J., Freschi, L., Alcalá-Franco, B., Facchini, M., Boyle, B., et al. (2015). Comparative genomics and biological characterization of sequential Pseudomonas aeruginosa isolates from persistent airways infection. BMC Genomics 16:1105. doi: 10.1186/s12864-015-2276-8

Chmiel, J. F., Berger, M., and Konstan, M. W. (2002). The role of inflammation in the pathophysiology of CF lung disease. Clin. Rev. Allergy Immunol. 23, 5-27. doi: 10.1385/CRIAI:23:1:005

Ciofu, O., Tolker-Nielsen, T., Jensen, P. Ø, Wang, H., and Høiby, N. (2015). Antimicrobial resistance, respiratory tract infections and role of biofilms in lung infections in cystic fibrosis patients. Adv. Drug Deliv. Rev. 85, 7-23. doi: 10.1016/j.addr.2014.11.017

Clark, S. T., Diaz Caballero, J., Cheang, M., Coburn, B., Wang, P. W., Donaldson, S. L., et al. (2015). Phenotypic diversity within a Pseudomonas aeruginosa population infecting an adult with cystic fibrosis. Sci Rep 5:10932. doi: 10.1038/ srep 10932

Cutting, G. R. (2015). Cystic fibrosis genetics: from molecular understanding to clinical application. Nat. Rev. Genet. 16, 45-56. doi: 10.1038/nrg3849

Davies, J., and O'Connor, S. (1978). Enzymatic modification of aminoglycoside antibiotics: 3-Nacetyltransferase with broad specificity that determines resistance to the novel aminoglycoside apramycin. Antimicrob. Agents Chemother. 14, 69-72. doi: 10.1128/AAC.14.1.69

European Medicines Agency [EMA] (2018). Reflection Paper on Use of Aminoglycosides in Animals in the European Union: Development of Resistance and Impact on Human and Animal Health. Amsterdam: EMA.

Folkesson, A., Jelsbak, L., Yang, L., Johansen, H. K., Ciofu, O., Høiby, N., et al. (2012). Adaptation of Pseudomonas aeruginosa to the cystic fibrosis airway: an evolutionary perspective. Nat. Rev. Microbiol. 10, 841-851. doi: 10.1038/ nrmicro2907

Forge, A., and Schacht, J. (2000). Aminoglycoside antibiotics. Audiol. Neurootol. $5,3-22$.

Gallant, C. V., Raivio, T. L., Olson, J. C., Woods, D. E., and Storey, D. G. (2000). Pseudomonas aeruginosa cystic fibrosis clinical isolates produce exotoxin A with altered ADP-ribosyltransferase activity and cytotoxicity. Microbiology (Reading) 146(Pt 8), 1891-1899. doi: 10.1099/00221287-146-8-1891

Hall-Stoodley, L., Costerton, J. W., and Stoodley, P. (2004). Bacterial biofilms: from the natural environment to infectious diseases. Nat. Rev. Microbiol. 2, 95-108. doi: $10.1038 /$ nrmicro821

Høiby, N., Ciofu, O., and Bjarnsholt, T. (2010). Pseudomonas aeruginosa biofilms in cystic fibrosis. Future Microbiol. 5, 1663-1674. doi: 10.2217/fmb.10.125

Ishikawa, M., García-Mateo, N., Čusak, A., López-Hernández, I., FernándezMartínez, M., Müller, M., et al. (2019). Lower ototoxicity and absence of hidden hearing loss point to gentamicin $\mathrm{Cla}$ and apramycin as promising antibiotics for clinical use. Sci. Rep. 9:2410. doi: 10.1038/s41598-019-38634-3

\section{SUPPLEMENTARY MATERIAL}

The Supplementary Material for this article can be found online at: https://www.frontiersin.org/articles/10.3389/fmicb. 2021.801152/full\#supplementary-material

Juhas, M., Widlake, E., Teo, J., Huseby, D. L., Tyrrell, J. M., Polikanov, Y. S., et al. (2019). In vitro activity of apramycin against multidrug-, carbapenemand aminoglycoside-resistant Enterobacteriaceae and Acinetobacter baumannii. J. Antimicrob. Chemother. 74, 944-952.

Jurado-Martín, I., Sainz-Mejías, M., and McClean, S. (2021). Pseudomonas aeruginosa: an audacious pathogen with an adaptable arsenal of virulence factors. Int. J. Mol. Sci. 22:3128. doi: 10.3390/ijms22063128

Kang, A. D., Smith, K. P., Berg, A. H., Truelson, K. A., Eliopoulos, G. M., McCoy, C., et al. (2018). Efficacy of apramycin against multidrug-resistant Acinetobacter baumannii in the murine neutropenic thigh model. Antimicrob. Agents Chemother. 62:e2585-17. doi: 10.1128/AAC.02585-17

Kang, A. D., Smith, K. P., Eliopoulos, G. M., Berg, A. H., McCoy, C., and Kirby, J. E. (2017). In vitro apramycin activity against multidrug-resistant Acinetobacter baumannii and Pseudomonas aeruginosa. Diagn. Microbiol. Infect. Dis. 88, 188-191. doi: 10.1016/j.diagmicrobio.2017.03.006

Kang, D., Zhang, L., and Kirienko, N. V. (2021). High-Throughput approaches for the identification of Pseudomonas aeruginosa antivirulents. mBio 12:e02240-20. doi: $10.1128 / \mathrm{mBio} .02240-20$

Keren, I., Kaldalu, N., Spoering, A., Wang, Y., and Lewis, K. (2004). Persister cells and tolerance to antimicrobials. FEMS Microbiol. Lett. 230, 13-18. doi: 10.1016/S0378-1097(03)00856-5

Li, Z., Kosorok, M. R., Farrell, P. M., Laxova, A., West, S. E., Green, C. G., et al. (2005). Longitudinal development of mucoid Pseudomonas aeruginosa infection and lung disease progression in children with cystic fibrosis. JAMA 293, 581-588. doi: 10.1001/jama.293.5.581

Lister, P. D., Wolter, D. J., and Hanson, N. D. (2009). Antibacterialresistant Pseudomonas aeruginosa: clinical impact and complex regulation of chromosomally encoded resistance mechanisms. Clin .Microbiol. Rev. 22, 582-610. doi: 10.1128/CMR.00040-09

Llanes, C., Köhler, T., Patry, I., Dehecq, B., van Delden, C., and Plésiat, P. (2011). Role of the MexEF-OprN efflux system in low-level resistance of Pseudomonas aeruginosa to ciprofloxacin. Antimicrob. Agents Chemother. 55, 5676-5684. doi: 10.1128/AAC.00101-11

Lovering, A. M., White, L. O., and Reeves, D. S. A. A. C. (1987). (1): a new aminoglycoside-acetylating enzyme modifying the $\mathrm{cl}$ aminogroup of apramycin. J. Antimicrob. Chemother. 20, 803-813. doi: 10.1093/jac/20. 6.803

Maiden, M. M., Hunt, A. M. A., Zachos, M. P., Gibson, J. A., Hurwitz, M. E., Mulks, M. H., et al. (2018). Triclosan is an aminoglycoside adjuvant for eradication of Pseudomonas aeruginosa biofilms. Antimicrob. Agents Chemother. 62:e0014618. doi: 10.1128/AAC.00146-18

Malhotra, S., Hayes, D. Jr., and Wozniak, D. J. (2019). Mucoid Pseudomonas aeruginosa and regional inflammation in the cystic fibrosis lung. J. Cyst. Fibros 18, 796-803. doi: 10.1016/j.jcf.2019.04.009

Malhotra, S., Limoli, D. H., English, A. E., Parsek, M. R., and Wozniak, D. J. (2018). Mixed communities of mucoid and nonmucoid Pseudomonas aeruginosa exhibit enhanced resistance to host antimicrobials. MBio 9::e00275-18. doi: 10.1128/mBio.00275-18

Masuda, N., Sakagawa, E., Ohya, S., Gotoh, N., Tsujimoto, H., and Nishino, T. (2000). Substrate specificities of MexAB-OprM, MexCD-OprJ, and MexXYoprM efflux pumps in Pseudomonas aeruginosa. Antimicrob. Agents Chemother. 44, 3322-3327. doi: 10.1128/AAC.44.12.3322-3327.2000

Matt, T., Ng, C. L., Lang, K., Sha, S. H., Akbergenov, R., Shcherbakov, D., et al. (2012). Dissociation of antibacterial activity and aminoglycoside ototoxicity in the 4-monosubstituted 2-deoxystreptamine apramycin. Proc. Natl. Acad. Sci. U.S.A. 109, 10984-10989. doi: 10.1073/pnas.1204073109

Meyer, M., Freihofer, P., Scherman, M., Teague, J., Lenaerts, A., and Böttger, E. C. (2014). In vivo efficacy of apramycin in murine infection models. Antimicrob. Agents. Chemother. 58, 6938-6941. doi: 10.1128/AAC.03239-14 
Michalska, M., and Wolf, P. (2015). Pseudomonas exotoxin A: optimized by evolution for effective killing. Front. Microbiol. 6:963. doi: 10.3389/fmicb.2015. 00963

Mogayzel, P. J. Jr., Naureckas, E. T., Robinson, K. A., Brady, C., Guill, M., Lahiri, T., et al. (2014). Cystic Fibrosis Foundation Pulmonary Clinical Practice Guidelines Committee. Cystic Fibrosis Foundation pulmonary guideline. pharmacologic approaches to prevention and eradication of initial Pseudomonas aeruginosa infection. Ann. Am. Thorac. Soc. 11, 1640-1650. doi: 10.1513/AnnalsATS. 201404-166OC

Morita, Y., Komori, Y., Mima, T., Kuroda, T., Mizushima, T., and Tsuchiya, T. (2001). Construction of a series of mutants lacking all of the four major mex operons for multidrug efflux pumps or possessing each one of the operons from Pseudomonas aeruginosa PAO1: MexCD-OprJ is an inducible pump. FEMS Microbiol. Lett. 202, 139-143. doi: 10.1111/j.1574-6968.2001.tb10794.x

National Committee for Clinical Laboratory Standards. (1999). Methods for Determining Bactericidal Activity of Antimicrobial Agents. Approved standard M26-A. Wayne, PA: NCCLS.

Pompilio, A., Savini, V., Fiscarelli, E., Gherardi, G., and Di Bonaventura, G. (2020). Clonal diversity, biofilm formation, and antimicrobial resistance among Stenotrophomonas maltophilia strains from cystic fibrosis and non-cystic fibrosis patients. Antibiotics (Basel) 9:15. doi: 10.3390/antibiotics9010015

Riedel, S., Vijayakumar, D., Berg, G., Kang, A. D., Smith, K. P., and Kirby, J. E. (2019). Evaluation of apramycin against spectinomycin-resistant and susceptible strains of Neisseria gonorrhoeae. J. Antimicrob. Chemother. 74, 1311-1316. doi: 10.1093/jac/dkz012

Rossi, E., La Rosa, R., Bartell, J. A., Marvig, R. L., Haagensen, J. A. J., Sommer, L. M., et al. (2021). Pseudomonas aeruginosa adaptation and evolution in patients with cystic fibrosis. Nat. Rev. Microbiol. 19, 331-342. doi: 10.1038/s41579-02000477-5

Smith, D. J., Lamont, I. L., Anderson, G. J., and Reid, D. W. (2013). Targeting iron uptake to control Pseudomonas aeruginosa infections in cystic fibrosis. Eur. Respir. J. 42, 1723-1736. doi: 10.1183/09031936.00124012

Sonnenwirth, A. C. (1980). "Stains and staining procedures," in Gradwohl's Clinical Laboratory Methods and Diagnosis, 8th Edn, eds A. C. Sonnenwirth and L. Jarett (St. Louis, MO: The C.V. Mosby Co), 380.

Stepanović, S., Vuković, D., Hola, V., Di Bonaventura, G., Djukić, S., Cirković, I., et al. (2007). Quantification of biofilm in microtiter plates: overview of testing conditions and practical recommendations for assessment of biofilm production by staphylococci. APMIS 115, 891-899. doi: 10.1111/j.1600-0463. 2007.apm_630.x

Tai, A. S., Sherrard, L. J., Kidd, T. J., Ramsay, K. A., Buckley, C., Syrmis, M., et al. (2017). Antibiotic perturbation of mixed-strain Pseudomonas aeruginosa infection in patients with cystic fibrosis. BMC Pulm. Med. 17:138. doi: 10.1186/ s12890-017-0482-7

The European Committee on Antimicrobial Susceptibility Testing (2021). Breakpoint Tables for Interpretation of MICs and Zone Diameters. Version 11.0. Växjö: The European Committee on Antimicrobial Susceptibility Testing.

Truelson, K. A., Brennan-Krohn, T., Smith, K. P., and Kirby, J. E. (2018). Evaluation of apramycin activity against methicillin-resistant, methicillinsensitive, and vancomycin-intermediate Staphylococcus aureus clinical isolates. Diagn. Microbiol. Infect. Dis. 92, 168-171. doi: 10.1016/j.diagmicrobio.2018. 05.018

Wachino, J., and Arakawa, Y. (2012). Exogenously acquired 16S rRNA methyltransferases found in aminoglycoside-resistant pathogenic Gramnegative bacteria: an update. Drug Resist. Updat. 15, 133-148. doi: 10.1016/j. drup.2012.05.001

Conflict of Interest: The authors declare that the research was conducted in the absence of any commercial or financial relationships that could be construed as a potential conflict of interest.

Publisher's Note: All claims expressed in this article are solely those of the authors and do not necessarily represent those of their affiliated organizations, or those of the publisher, the editors and the reviewers. Any product that may be evaluated in this article, or claim that may be made by its manufacturer, is not guaranteed or endorsed by the publisher.

Copyright (C) 2022 Di Bonaventura, Lupetti, Verginelli, Giancristofaro, Barbieri, Gherardi and Pompilio. This is an open-access article distributed under the terms of the Creative Commons Attribution License (CC BY). The use, distribution or reproduction in other forums is permitted, provided the original author(s) and the copyright owner(s) are credited and that the original publication in this journal is cited, in accordance with accepted academic practice. No use, distribution or reproduction is permitted which does not comply with these terms. 